\title{
Effects of different anesthesia methods on postoperative transient neurological syndrome in patients with lumbar disc herniation
}

\author{
SHUNHONG MAO, CHUNHUA ZHU and YULIN CHANG \\ Department of Anesthesiology, Cangzhou Central Hospital, Cangzhou, Hebei 061000, P.R. China
}

Received May 11, 2017; Accepted August 2, 2017

DOI: $10.3892 /$ etm.2017.4900

\begin{abstract}
The objective of the present study was to investigate the effects of different anesthesia methods on postoperative transient neurologic syndrome (TNS) in patients with lumbar disc herniation (LDH). Ninety-six patients with LDH were selected from November 2015 to October 2016 in Cangzhou Central hospital. All patients were treated with percutaneous transforaminal endoscopic discectomy. The patients were randomly divided into the control group and observation group, with 48 patients each. Combined spinal-epidural anesthesia was performed for patients in the control group, while epidural anesthesia was applied in the observation group. The levels of $\mathrm{T}$ lymphocyte subsets $\left(\mathrm{CD}^{+}\right.$and $\left.\mathrm{CD}^{+}\right)$and inflammatory factors (IL-2 and TNF- $\alpha$ ) were measured and compared before and 1 week after surgery. The incidence rate of TNS within 1 week after surgery was compared between the two groups. Fugl-Meyer Assessment was used to evaluate lower limb motor function and sensory disturbances at 1,3 and 5 days after treatment. One week after treatment, the serum levels of $\mathrm{CD}^{+}$and $\mathrm{CD}^{+}$in the two groups were significantly lower than those before surgery $(p<0.05)$, but no significant differences were found between the groups $(p>0.05)$. The incidence rate of TNS within 1 week after surgery was significantly lower in the observation group than in the control group $(\mathrm{p}<0.05)$. The scores of lower limb motor function and sensory disturbances in the observation group evaluated at 1, 3 and 5 days after treatment were significantly higher than those in the control group $(\mathrm{p}<0.05)$. In conclusion, combined spinal-epidural anesthesia and epidural anesthesia caused no significant differences in immune function or inflammatory indexes in patients with LDH. However, the application of epidural anesthesia significantly reduced the incidence rate of postoperative TNS, which in turn reduced nerve damage.
\end{abstract}

Correspondence to: Dr Shunhong Mao, Department of Anesthesiology, Cangzhou Central Hospital, 16 Xinhua West Road, Yunhe, Cangzhou, Hebei 061000, P.R. China

E-mail: shunhong_mao@hotmail.com

Key words: percutaneous transforaminal endoscopic discectomy, anesthesia, lumbar disc herniation, transient neurologic syndrome

\section{Introduction}

Lumbar disc herniation (LDH) is a common orthopedic disease characterized by low back pain and radiating pain, and has become a global health issue (1). Treatment of LDH may include methods such as conservative treatment, interventional therapy and surgical treatment, although percutaneous transforaminal endoscopic discectomy (PTED) is clinically preferred (2). Intravertebral anesthesia is generally used in PTED. Intravertebral anesthesia, which includes epidural anesthesia, spinal anesthesia, spinal anesthesia combined epidural anesthesia, and sacral anesthesia, is a method of local anesthesia (3). Compared with general anesthesia and the use of intravenous opioids, intravertebral anesthesia can more accurately control surgical or postoperative pain with the use of local anesthetics for nerve block, which is beneficial for the recovery of the patient's body function. However, the potential neurological complications from intravertebral anesthesia have attracted increasing attention (4). Neurological complications of intravertebral anesthesia include transient neurologic syndrome (TNS), cauda equina syndrome and permanent lumbar radiculopathy. Different from cauda equina syndrome, most patients with TNS have certain motor dysfunctions and sensory disturbances. However, nuclear magnetic resonance and electrophysiological examination generally show no abnormalities, which is a challenge in clinical practice $(5,6)$. In this study, patients with LDH were treated with combined spinal-epidural anesthesia or epidural anesthesia. The incidence of postoperative TNS was also observed.

\section{Patients and methods}

General information. From November 2015 to October 2016, 96 patients with LDH were selected in Cangzhou Central hospital. All patients were treated with PTED. The patients were randomly divided into the control group and observation group, with 48 patients each. Inclusion criteria: i) Patients with LDH diagnosed by imaging and discography; ii) patients with varying degrees of low back pain, and positive femoral nerve or sciatic nerve stretching test; and iii) patients or their family members signed the informed consent. Exclusion criteria: Patients with severe osteomalacia, osteoporosis, malignant tumors, severe cardiovascular and cerebrovascular diseases or liver and kidney dysfunction. There were no 
significant differences in the baseline parameters between the two groups $(\mathrm{p}>0.05)$ (Table I).

Anesthesia. All the patients were subjected to intramuscular injection of atropine $(0.5 \mathrm{mg}$, SFDA approval no. H4102367; Anyang Jiuzhou Pharmaceutical Co., Ltd., Henan, China). Routine monitoring of changes in $\mathrm{SpO}_{2}$, breathing, pulse and blood pressure were performed while patients were in the prone position. Patients in the observation group received epidural anesthesia. The intervertebral space at the top of the lesion area was used to place the epidural tube. Next, a $5 \mathrm{ml}$ mixture of $1 \%$ lidocaine (SFDA approval no. H14023559; Jincheng Haisi Pharmaceutical Co., Ltd., Jincheng, China) and $0.375 \%$ ropivacaine (SFDA approval no. H20050325; China Resources Pharmaceutical Group Ltd., Beijing, China) was injected. If no adverse reactions occurred, an additional $10 \mathrm{ml}$ of the mixture was injected with the plane controlled below T6-T8. Patients in the control group were treated with combined spinal-epidural anesthesia. The area between L2 and L3 was punctured to place the spinal needle. Clear cerebrospinal fluid outflow indicated smooth backflow. At this point, $2 \mathrm{ml}$ of $1 \%$ ropivacaine was injected within $15 \mathrm{sec}$. The spinal needle was then withdrawn, and an epidural tube was inserted 4-cm long towards the head end. With patients in the supine position, the plane was adjusted to T6. Epidural analgesia was administered to both groups of patients using $0.2 \mu \mathrm{g} / \mathrm{ml}$ sufentanil (SFDA approval no. H20054172; Yichang Humanwell Pharmaceutical Co., Ltd., Yichang, China) and $0.12 \%$ ropivacaine with infusion rate of $5 \mathrm{ml} / \mathrm{h}$. The epidural catheter was removed when platelet density was $>100,000 / \mathrm{mm}^{3}$, international normalized ratio was $<1.5$ and clotting time returned to normal.

Index detection. Venous blood samples $(5 \mathrm{ml})$ were collected from the two groups of patients before and 7 days after surgery (fasting for at least $8 \mathrm{~h}$ ), and serum was separated and extracted by centrifugation (Shenzhen Chaojie Experimental Instrument Co., Ltd., Shenzhen, China). After, rabbit monoclonal CD4 (dilution, 1:50; cat. no. 100405) and rabbit monoclonal CD8 antibody (dilution, 1:50; cat. no. 100706), purchased from BioLegend (San Diego, CA, USA), were added and incubated at $4^{\circ} \mathrm{C}$ for $30 \mathrm{~min}$ in the dark. Flow cytometry (BD Biosciences, Franklin Lakes, NJ, USA) was used to detect the levels of $\mathrm{CD}^{+}$and $\mathrm{CD}^{+}$cells. The serum levels of interleukin-2 (IL-2) and tumor necrosis factor- $\alpha$ (TNF- $\alpha$ ) were determined using an enzyme-linked immunosorbent assay (ELISA) kit according to the manufacturer's instructions (Thermo Fisher Scientific, Inc., Waltham, MA, USA). A total of $50 \mu \mathrm{l}$ of standards and serum were added to each well of the reaction plate, and $100 \mu \mathrm{l}$ of the enzyme-labeled solution was added to each well. After incubation at $37^{\circ} \mathrm{C}$ for $1 \mathrm{~h}$, plates were washed 5 times. After, chromogenic agent solutions A and B (50 $\mu \mathrm{l}$ each) were added and incubated at room temperature in the dark for $15 \mathrm{~min}$. Next, $50 \mu \mathrm{l}$ of the stop solution was added. Optical density (OD) values were measured at $450 \mathrm{~nm}$ using a microplate reader (Potebio, Jiangsu, China) within $15 \mathrm{~min}$ to calculate the concentrations of IL-2 and TNF- $\alpha$.

Evaluation criteria. Fasting venous blood samples $(5 \mathrm{ml})$ were collected from patients before and 7 days after surgery to prep-
Table I. Comparison of baseline parameters between the two groups of patients.

\begin{tabular}{lcccc}
\hline & $\begin{array}{c}\text { Observation } \\
\text { group } \\
(\mathrm{n}=48)\end{array}$ & $\begin{array}{c}\text { Control } \\
\text { group } \\
(\mathrm{n}=48)\end{array}$ & $\mathrm{t} / \chi^{2}$ & P-value \\
\hline Items & $27 / 21$ & $25 / 23$ & 0.168 & 0.682 \\
Sex (male/female) & $40-68$ & $40-65$ & & \\
Age (years) & $49.56 \pm 4.42$ & $49.85 \pm 3.53$ & 0.355 & 0.723 \\
$\begin{array}{l}\text { Average age } \\
\text { (years) }\end{array}$ & & & & \\
$\begin{array}{l}\text { Course of disease } \\
\text { (months) }\end{array}$ & $18.23 \pm 4.75$ & $18.37 \pm 4.38$ & 0.150 & 0.881 \\
$\begin{array}{l}\text { Types (n, \%) } \\
\text { Central type }\end{array}$ & $14(29.17)$ & $16(33.33)$ & & \\
$\begin{array}{l}\text { Paracentral type } \\
\text { L4-L5 far }\end{array}$ & $19(39.58)$ & $21(43.75)$ & 0.849 & 0.654 \\
lateral type & $15(31.25)$ & $11(22.92)$ & & \\
\hline
\end{tabular}

are serum samples. The levels of $\mathrm{CD}^{+}$and $\mathrm{CD} 8^{+} \mathrm{T}$ lymphocyte subsets were measured by flow cytometry. Serum IL-2 and TNF- $\alpha$ concentrations were measured by ELISA.

Lower limb motor function was scored according to Fugl-Meyer Assessment (FMA) (7) at 1, 3 and 5 days after surgery. Cooperative motion of lower limb flexor and extensor, reflection activity, coordination ability and speed, and separating motion were included in the scoring system. A total of 0 points indicated that movement could not be carried out, and 2 points indicated that movement could be fully completed. The total score was 34 points. A score of $<32$ indicated movement disorder, and scores were negatively correlated with movement disorder. Lower limb sensory scoring was performed according to FMA, and pain, temperature, and touch sensation were included to evaluate sensory disturbance. A total of 0 points indicated no sensation, and 2 points represented normal sensation. The total score was 20 points, and score and sensory disorder were negatively correlated.

Determination of TNS. After surgery, patients showed unilateral or bilateral lower limb movement disorders, sensory disturbances, burning pain and squeezing or radiating pain. If electrophysiological examination showed no abnormalities, the patients were diagnosed with TNS. According to the severity, TNS was divided into different degrees: i) Level I: Obvious unilateral or bilateral movement disorder and sensory disturbance, movement disorder score $<26$ points, and sensory disturbance score $<16$; ii) Level II: No obvious unilateral or bilateral movement disorder, score $\geq 26$ points, obvious sensory disturbances and sensory disturbance score $<16$; and iii) no obvious unilateral or bilateral movement disorder and sensory disturbance, and only the presence of numbness and pain.

Statistical analysis. Data were analyzed using SPSS 19.0 statistical software (SPSS Inc., Chicago, IL, USA). Numerical data are presented as mean \pm standard deviation (mean \pm SD), and paired t-test was used for intragroup and intergroup comparisons. Categorical data are presented as rate, and were 


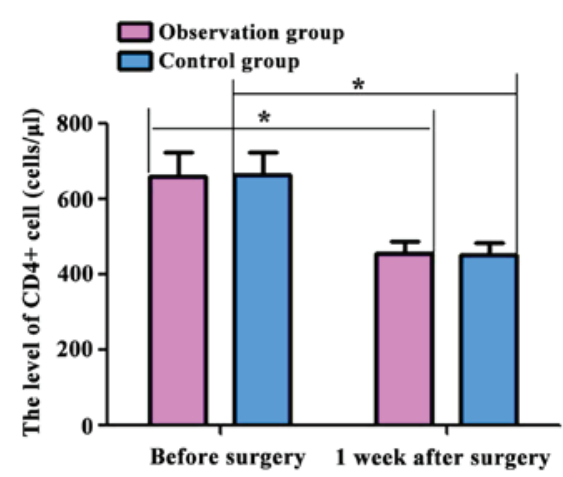

Figure 1. Comparison of $\mathrm{CD}^{+}$levels in the two groups of patients before and 1 week after surgery. Flow cytometry showed that the postoperative $\mathrm{CD}^{+}$levels in the two groups were significantly lower than those preoperatively $(\mathrm{p}<0.05)$. No significant differences were found in the preoperative and postoperative levels between the two groups ( $>>0.05$ ). Compared with preoperative levels, ${ }^{*} \mathrm{p}<0.05$.

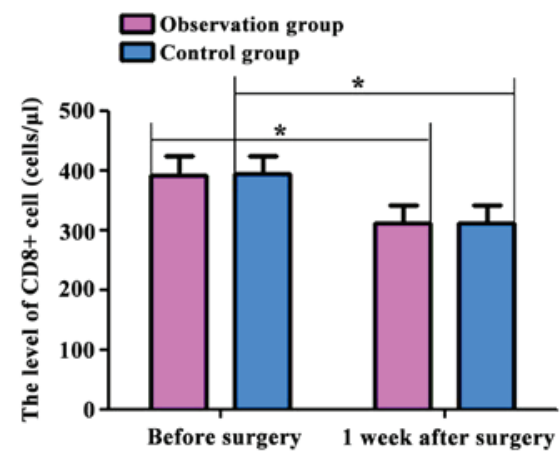

Figure 2. Comparison of $\mathrm{CD}^{+}$levels in the two groups of patients before and 1 week after surgery. Flow cytometry showed that the postoperative $\mathrm{CD} 8^{+}$levels in the two groups were significantly lower than those preoperatively $(\mathrm{p}<0.05)$. No significant differences were found in the preoperative and postoperative levels between the two groups ( $p>0.05)$. Compared with preoperative levels, ${ }^{*} \mathrm{p}<0.05$.

tested by $\chi^{2}$ test. $\mathrm{P}<0.05$ was considered to indicate a statistically significant difference.

\section{Results}

The levels of T lymphocyte subsets and inflammatory factors in the two groups. The levels of $\mathrm{T}$ lymphocyte subsets $\left(\mathrm{CD} 4^{+}\right.$ and $\mathrm{CD}^{+}$) and inflammatory factors (IL-2 and TNF- $\alpha$ ) in the two groups were compared. Before and 1 week after surgery, $\mathrm{CD} 4^{+}$levels were: $662.54 \pm 62.54,457.36 \pm 47.48$ cells $/ \mu 1$; CD8 ${ }^{+}$levels were: $393.58 \pm 43.45,313.74 \pm 37.52$ cells $/ \mu 1$; IL-2 levels were: $8.43 \pm 2.37,15.68 \pm 3.26 \mathrm{pg} / \mathrm{ml}$; and TNF- $\alpha$ levels were: $9.47 \pm 3.54,28.67 \pm 3.43 \mathrm{pg} / \mathrm{ml}$ in the observation group. At the same time-points, $\mathrm{CD} 4^{+}$levels were: $665.36 \pm 69.36,453.46 \pm 42.46$ cells $/ \mu 1 ; \mathrm{CD}^{+}$levels were: $394.74 \pm 42.48,312.75 \pm 35.47$ cells $/ \mu 1$; IL-2 levels were: $8.63 \pm 2.48,15.27 \pm 3.23 \mathrm{pg} / \mathrm{ml}$; TNF- $\alpha$ levels were: $9.38 \pm 3.17$, $27.85 \pm 3.52 \mathrm{pg} / \mathrm{ml}$ in the control group. The serum levels of $\mathrm{CD}^{+}$and $\mathrm{CD} 8^{+}$cells in the two groups were decreased, and the levels of IL-2 and TNF- $\alpha$ were increased at 7 days after surgery compared with those before surgery $(\mathrm{p}<0.05)$. However, no significant differences were found between the two groups ( $>0.05$ ) (Figs. 1-4).



Figure 3. Comparison of IL-2 levels in the two groups of patients before and 1 week after surgery. Postoperative IL-2 levels in the two groups were significantly higher than those preoperatively $(\mathrm{p}<0.05)$. No significant differences were found in the preoperative and postoperative levels between two groups $(p>0.05)$. Compared with preoperative levels, ${ }^{*} p<0.05$. IL, interleukin.

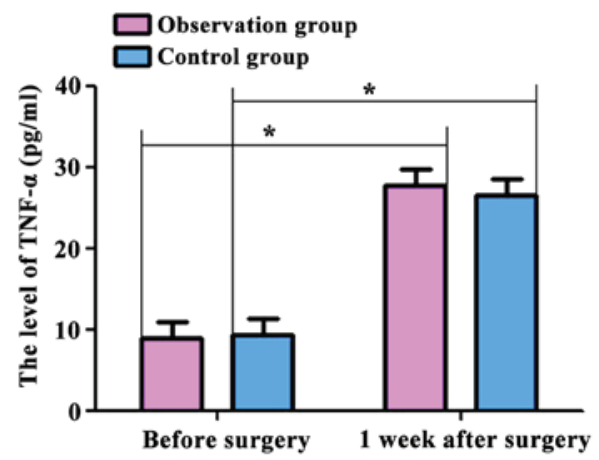

Figure 4. Comparison of TNF- $\alpha$ levels in the two groups of patients before and 1 week after surgery. Postoperative TNF- $\alpha$ levels in the two groups were significantly higher than those preoperatively $(\mathrm{p}<0.05)$. No significant differences were found in the preoperative and postoperative levels between the two groups $(\mathrm{p}>0.05)$. Compared with preoperative levels, ${ }^{*} \mathrm{p}<0.05$. TNF- $\alpha$, tumor necrosis factor- $\alpha$.

The incidence rates of TNS within 1 week after surgery. The incidence rates of TNS within 1 week after surgery were compared between the two groups. The incidence rate of TNS in the observation group was significantly lower than that in the control group $(\mathrm{p}<0.05)$ (Table II).

Lower limb movement disorder scores between the two groups. Lower limb movement disorder scores were compared between the two groups. The scores in the observation group were significantly higher than those in the control group at 1,3 and 5 days after surgery $(\mathrm{p}<0.05)$ (Table III).

Sensory disorder scores between the two groups. Sensory disorder scores were compared between the two groups. The scores in the observation group were significantly higher than those in the control group at 1, 3 and 5 days after surgery $(p<0.05)($ Table IV).

\section{Discussion}

LDH is the herniation of the nucleus pulposus caused by changes of water molecules in the nucleus pulposus of the intervertebral disc and the prominent nucleus pulposus can 
Table II. Comparison of the incidence rates of TNS between the two groups $(\mathrm{n}, \%)$.

\begin{tabular}{|c|c|c|c|c|c|}
\hline Groups & Cases & Level I & Level II & Level III & $\begin{array}{c}\text { Incidence } \\
\text { rates of } \\
\text { TNS }\end{array}$ \\
\hline Observation & 48 & $1(2.08)$ & $2(4.17)$ & $3(6.25)$ & $6(12.50)$ \\
\hline Control & 48 & $4(8.33)$ & $5(10.42)$ & 7 (14.58) & $16(33.33)$ \\
\hline$\chi^{2}$ & & & & & 4.775 \\
\hline P-value & & & & & 0.029 \\
\hline
\end{tabular}

TNS, transient neurologic syndrome.

Table III. Comparison of lower limb movement disorder scores between the two groups.

\begin{tabular}{lcccc}
\hline Groups & Cases & 1 day & 3 days & 5 days \\
\hline Observation & 48 & $28.95 \pm 1.14$ & $29.36 \pm 1.13$ & $31.13 \pm 1.12$ \\
Control & 48 & $26.16 \pm 1.23$ & $27.73 \pm 1.25$ & $29.26 \pm 1.17$ \\
t-test & & 11.526 & 6.702 & 7.999 \\
P-value & & $<0.001$ & $<0.001$ & $<0.001$ \\
\hline
\end{tabular}

Table IV. Comparison of sensory disorder scores between the two groups.

\begin{tabular}{lcccc}
\hline Groups & Cases & 1 day & 3 days & 5 days \\
\hline Observation & 48 & $16.84 \pm 2.34$ & $17.36 \pm 2.33$ & $19.53 \pm 1.22$ \\
Control & 48 & $15.76 \pm 2.25$ & $16.43 \pm 2.15$ & $18.96 \pm 1.27$ \\
t-test & & 2.305 & 2.032 & 2.242 \\
P-value & & 0.023 & 0.045 & 0.027 \\
\hline
\end{tabular}

oppress the cauda equina or nerve root to produce pain. LDH mostly occurs in the L4/L5 segment (8). Currently, PTED is widely used in clinical practice. By precise puncture and positioning, the lesion can be reached through intervertebral foramen puncture to effectively reduce the pain of patients. In addition, intravertebral anesthesia is performed before surgery. Conscious patients will maintain the function of motor and tactile nerves. Through communication with patients, nerve root damage can be avoided, thereby reducing pain $(9,10)$. Surgical trauma, the nervousness of patients and narcotic drugs can cause a stress response, which in turn inhibits immune function. Therefore, immune dysfunction and associated inflammatory responses will occur (11). Stress responses can lead to increased secretion of adrenaline and catechol, resulting in abnormal $\mathrm{CD}^{+}$and $\mathrm{CD}^{+}$levels. Therefore, immune function disorders will occur and serum levels of IL-2 and TNF- $\alpha$ will increase to induce inflammation $(12,13)$. In this study, serum $\mathrm{CD}^{+}$and $\mathrm{CD}^{+}$levels decreased, and IL-2 and TNF- $\alpha$ levels increased at 1 week after treatment compared with preoperative levels. Moreover, there were no significant differences between the two groups $(p>0.05)$. These obser- vations can be explained by surgical trauma and anesthesia stimulation. However, combined spinal-epidural anesthesia and epidural anesthesia can cause immune dysfunction and inflammation within a short time period after treatment, and there are no essential differences between them.

Related studies reported that the incidence rate of TNS was $3-33 \%$, which may represent mild toxicity from local anesthetics (14). Results from this study showed that TNS was observed in both groups of patients within 1 week after surgery, and the incidence rate of TNS in the observation group was significantly lower than that in the control group $(\mathrm{p}<0.05)$. Puncture at the dura of the spinal nerve root, drug injection, and catheterization were performed in both combined spinal-epidural anesthesia and epidural anesthesia. The operations can cause nerve root injury and anesthetic can easily accumulate and precipitate at the dura of the spinal nerve root to achieve nerve block function. Additionally, the intrathecal vulnerable area is the spinal nerve root reaching the spinal cord, and the nerve fibers are unmyelinated. After local anesthetic injection, anesthetic will spread to the spinal nerve root. In addition, excessive air or salt water will also be injected to increase the pressure within the spinal canal, which in turn oppresses nerves and induces nerve damage $(15,16)$. In addition, puncture is generally performed before the complete drying of disinfection liquid. Therefore, disinfector can easily spread to the spinal canal to damage nerve roots. Compared with epidural anesthesia, operations using combined spinal-epidural anesthesia can cause more severe soft tissue and nerve root injuries (17).

In this study, patients in both groups showed lower limb movement disorder and sensory disturbances, and the scores in the observation group were significantly higher than those in the control group at 1,3 and 5 days after surgery $(\mathrm{p}<0.05)$. This was because all local anesthetics are neurotoxic, and repeated injection can cause high local concentrations. The surface active molecules will aggregate in biofilms to affect the structures of proteins and phospholipids on nerve fiber membranes, resulting in irreversible damage (18). Similarly, local anesthetics cause increased intracellular calcium concentrations, and higher calcium concentrations cause greater damage to nerve cells (19). Long-term analgesia catheterization can lead to long-term exposure of local nerves to drugs, as well as spinal cord tissue edema. Therefore, patients will present with lower limb numbness, weakness, pain and other symptoms. In combined spinal-epidural anesthesia, local anesthetics are injected directly into the cerebrospinal fluid to act on anterior and posterior roots of the spinal nerve and spinal cord, which in turn lead to even higher local drug concentrations and spinal nerve toxicity. Therefore, nerve damage caused by this method is more severe than that caused by epidural anesthesia alone (20).

The safety of intravertebral anesthesia used in the surgical treatment of patients with LDH should be recognized, and this method should not be ignored because of the occurrence of TNS. Proper clinical prevention should be applied to reduce the incidence rate of TNS. Comprehensive assessment should be performed before surgery to determine the application of intravertebral anesthesia. With comprehensive assessment, the use of epidural anesthesia is encouraged. In the case of puncture difficulty, repeated puncture should be avoided to reduce 
damage of the penetration barrier caused by anesthetics. Soft and effective non-invasive catheters should be used as epidural catheters. The dose of anesthetics should also be controlled. The plane of anesthesia should be controlled in a relatively wide range to avoid excessive concentrations of local anesthetics.

In conclusion, to reduce the incidence rate of TNS and increase the safety of surgery, epidural anesthesia, a form of intravertebral anesthesia, should be applied in the PTED treatment of patients with LDH.

\section{References}

1. Lurie JD, Tosteson TD, Tosteson AN, Zhao W, Morgan TS, Abdu WA, Herkowitz H and Weinstein JN: Surgical versus nonoperative treatment for lumbar disc herniation: Eight-year results for the spine patient outcomes research trial. Spine 39: 3-16, 2014

2. Sinkemani A, Hong X, Gao ZX, Zhuang SY, Jiang ZL, Zhang SD, Bao JP, Zhu L, Zhang P, Xie XH, et al: Outcomes of microendoscopic discectomy and percutaneous transforaminal endoscopic discectomy for the treatment of lumbar disc herniation: A comparative retrospective study. Asian Spine J 9: 833-840, 2015

3. Malvasi A, Tinelli A, Brizzi A, Guido M,Laterza F, De Nunzio G, Bochicchio M, Ghi T, Stark M, Benhamou D, et al: Intrapartum sonography head transverse and asynclitic diagnosis with and without epidural analgesia initiated early during the first stage of labor. Eur Rev Med Pharmacol Sci 15: 518-523, 2011.

4. Nash DM, Mustafa RA, McArthur E, Wijeysundera DN, Paterson JM, Sharan S, Vinden C, Wald R, Welk B Sessler DI, et al: Combined general and neuraxial anesthesia versus general anesthesia: A population-based cohort study. Can J Anaesth 62: 356-368, 2015.

5. Talakoub R, Golparvar M and Arshi R: The effect of early ambulation on the incidence of neurological complication after spinal anesthesia with lidocaine. J Res Med Sci 20: 383-386, 2015.

6. Rastogi S, Bhandari R, Sharma V and Pandey T: Neurological complications following spinal anaesthesia in a patient with congenital absence of lumbar vertebra. Indian J Anaesth 58 484-486, 2014

7. Wei XJ, Tong KY and Hu XL: The responsiveness and correlation between Fugl-Meyer Assessment, Motor Status Scale, and the Action Research Arm Test in chronic stroke with upper-extremity rehabilitation robotic training. Int $\mathrm{J}$ Rehabil Res 34: 349-356, 2011.
8. Chadha M, Sharma G, Arora SS and Kochar V: Association of facet tropism with lumbar disc herniation. Eur Spine J 22: 1045-1052, 2013.

9. Sawai T, Nakahira J and Minami T: Paraplegia caused by giant intradural herniation of a lumbar disk after combined spinal-epidural anesthesia in total hip arthroplasty. J Clin Anesth 32: 169-171, 2016.

10. Vadi MG, Patel N and Stiegler MP: Local anesthetic systemic toxicity after combined psoas compartment-sciatic nerve block: Analysis of decision factors and diagnostic delay. Anesthesiology 120: 987-996, 2014.

11. Pearson AM and Lurie JD: Surgical versus nonoperative treatment: How do we choose the right approach to lumbar disk herniation? Pain Manag 4: 247-249, 2014.

12. Feng Q, Wei H, Morihara J, Stern J, Yu M, Kiviat N, Hellstrom I and Hellstrom KE: Th2 type inflammation promotes the gradual progression of HPV-infected cervical cells to cervical carcinoma. Gynecol Oncol 127: 412-419, 2012.

13. DeBerge MP, Ely KH and Enelow RI: Soluble, but not transmembrane, TNF- $\alpha$ is required during influenza infection to limit the magnitude of immune responses and the extent of immunopathology. J Immunol 192: 5839-5851, 2014.

14. Etezadi F, Karimi YK, Ahangary A, Shokri H, Imani F, Safari S and Khajavi MR: The effect of needle type, duration of surgery and position of the patient on the risk of transient neurologic symptoms. Anesth Pain Med 2: 154-158, 2013.

15. Goeller JK, Joselyn A, Martin DP, Bhalla T, Dairo O, Herz DB, Alpert SA and Tobias JD: Epidural pressure changes following caudal blockade: A prospective, observational study. J Anesth 30: 578-582, 2016.

16. Tsui BC: Epidural and caudal anesthesia. In: Pediatric Atlas of Ultrasound- and Nerve Stimulation-Guided Regional Anesthesia. Tsui BC and Suresh S (eds). Springer, New York, NY, pp495-525, 2016

17. Kato J, Konishi J, Yoshida H, Furuya T, Kashiwai A, Yokotsuka S, Gokan D and Ogawa S: Cauda equina syndrome following combined spinal and epidural anesthesia: A case report. Can J Anaesth 58: 638-641, 2011.

18. Wang Z, Shen J, Wang J, Lu T, Li C, Zhang X, Liu L and Ding Z: Lithium attenuates bupivacaine-induced neurotoxicity in vitro through phosphatidylinositol-3-kinase/threonine-serine protein kinase B- and extracellular signal-regulated kinase-dependent mechanisms. Neuroscience 206: 190-200, 2012.

19. Lin EP, Soriano SG and Loepke AW: Anesthetic neurotoxicity. Anesthesiol Clin 32: 133-155, 2014.

20. Yun MJ, Kwon MY, Kim DH, Lee JW, Paterson JM, Sharan S, Vinden $\mathrm{C}$ and Wald R: Combined spinal-epidural anesthesia using a reduced-dose of spinal bupivacaine and epidural top up leads to faster motor recovery after lower extremity surgeries. Korean J Anesthesiol 66: 28-33, 2014. 